\title{
Debonding detection of FRP strengthened concrete beams by using impedance measurements and an ensemble PSO adaptive spectral model
}

\author{
Rui Sun, Enrique Sevillano, Ricardo Perera
}

\begin{abstract}
A B S T R A C T
An impedance-based midspan debonding identification method for RC beams strengthened with FRP strips is presented in this paper using piezoelectric ceramic (PZT) sensor-actuators. To reach this purpose, firstly, a two-dimensional electromechanical impedance model is proposed to predict the electrical admittance of the PZT transducer bonded to the FRP strips of an RC beam. Considering the impedance is measured in high frequencies, a spectral element model of the bonded-PZT-FRP strengthened beam is developed. This model, in conjunction with experimental measurements of PZT transducers, is used to present an updating methodology to quantitatively detect interfacial debonding of these kinds of structures. To improve the performance and accuracy of the detection algorithm in a challenging problem such as ours, the structural health monitoring approach is solved with an ensemble process based on particle of swarm. An adaptive mesh scheme has also been developed to increase the reliability in locating the area in which debonding initiates. Predictions carried out with experimental results have showed the effectiveness and potential of the proposed method to detect prematurely at its earliest stages a critical failure mode such as that due to midspan debonding of the FRP strip.
\end{abstract}

\section{Introduction}

Although the strengthening of concrete structures with Fibre Reinforced Polymer (FRP) composites is becoming more and more extended, the premature debonding brittle failure is, without a doubt, an important disadvantage of this technique. A typical dominant failure mode such as intermediate crack (IC) debonding, initiated at a major flexural crack and then propagated towards a plate end, is an example of this [1-3]. Although many works have been published in the last years about experimental study and computational modeling of the debonding phenomenon, little work has been developed up to date on bond health monitoring and the initiation of debonding at a very early stage, long before a structure fails, in spite of its importance. Therefore, methodologies able to monitor the long-term efficiency of bonding and successfully identify the initiation of FRP debonding constitute a challenge to be met.

Structural health monitoring systems have been proposed for this purpose based on strain tracking. One of the main disadvantages of this approach is due to the need to use expensive finite element models to carry out the procedure, able to define the stage of the concrete/composite bond from the measured strain distribution. However, although some attempts have been made recently to eliminate this shortcoming [4], detection techniques based on strains are also restrained by their limited sensitivity to identify minor defects such as those that might originate the debonding failure. Furthermore, the use of controlled load tests or monitoring the traffic while the structure is in operation to obtain suitable strain profiles increase the complexity of the damage identification procedure.

Other techniques, such as acoustic emission have also been used in the past $[5,6]$. The detection of new defects or the propagation of existing defects requires continuous monitoring of the structure under evaluation and involves the storage and management of a huge amount of data. Furthermore, considering that acoustic emission is a passive non-destructive technique other difficulties arise from the inability of vehicular traffic to generate a meaningful response from the structure and from the need to filter out noise.

One very promising active non-destructive evaluation method for this particular application is impedance-based structural health monitoring (SHM) using piezoelectric ceramic (PZT) sensoractuators [7]. The electrical impedance of the PZT can be directly related to the mechanical impedance of the host structural component where the PZT transducers are attached. Since the 
structural mechanical impedance will be affected by the presence of structural damage, comparisons of admittance (inverse of impedance) spectra at various times during the service period of the structure can be used as damage indicator. Any change in the spectra might be an indication of a change in the structural integrity.

The electrical impedance is measured at high frequencies with which this methodology appears to be very sensitive to incipient damage in structural systems as desired for our application. Furthermore, due to their size and lightness PZT sensors are nonintrusively attached and do not affect the dynamic response of the structure. Additionally, this strategy does not need permanent monitoring since damage is detected by periodically checking the structural health.

Up to now, the impedance method has been used in numerous applications to detect damage. Experimental assessments were implemented by researchers to investigate the dynamic characteristics of structure and identify the severity and location of damages. Min et al. [8] deployed an impedance-based SHM technique for a bolt-jointed aluminium beam, a pipe system and a real-scale bridge for damage detection, by using the experimental data to train a neural network (NN). Giurgiutiu et al. [9] employed impedance technique to monitor the healthy condition of spot-welded structural joints for the first time, and this approach was applied on aging aircraft structures [10]. The evaluation of the debonding condition of a carbon fiber reinforced polymer (CFRP) strengthened concrete beam was performed by Park et al. [11] using an electromechanical wireless SHM technique. Structural crack damage was identified by Xu et al. [12] using impedance analysis.

Numerical simulation has also drawn researchers' attention in this field. A one-dimensional electro-mechanical impedance (EMI) model was firstly established by Liang et al. [13], where the analytical formulation of PZT impedance was given; it can be observed from this model that the electrical impedance of PZT is associated not only with the electrical properties of PZT, but also with the mechanical impedance of PZT patch and host structure. Other numerical approaches were carried out by Kuang et al. [14], Yan et al. [15,16] and Tseng et al. [17] among others.

Finite element models have been developed in the past to obtain the electro-mechanical impedance of different kinds of structures $[17,18]$. In all cases, the mesh size must be smaller than the calculated wavelength related to the frequency sweep range; however, it leads to a huge number of elements and high computational cost. To overcome this disadvantage, spectral element method (SEM) provides reasonable accuracy in resolving dynamic characteristics even at high frequency with very limited elements in contrast to the conventional finite elements. By applying discrete Fourier transform (DFT), the dynamic equations are transformed into the frequency domain and, therefore, few elements are required, resulting in an enormous reduction of computational resources [19-22]. This makes SEM to be a very suitable approach to capture the impedance spectra of bonded PZT patch which involves a high frequency range.

To the author's knowledge, only a few works are focused on the prediction of damage detection in FRP strengthened RC beams using EMI [23-26] and practically none takes advantage of combining experimental tests with numerical modeling. Experimental studies allow basically a qualitative damage identification by comparing further sets of data to a set of baseline impedance data. However, an efficient numerical model might provide additional information contributing to a more accurate description of damage quantification and location and, furthermore, it might also be useful for the suitable design of a PZT SHM system.

In the present work, debonding detection in RC beams externally flexural strengthened with FRP composites using the EMI technique is explored. For this, a model updating procedure will be developed. To make the proposed methodology successful one of the main objectives is the implementation of a bonded-PZT-FRP spectral beam element to be used in the calculation of the electrical admittance of the PZT transducers bonded to the structure. The use of the finite element method in a high frequency SHM approach, such as impedance-based method, would require a high number of elements of small size with an enormous expense in computation time and effort since the element size has to be comparable to wavelengths, which are very small in high frequencies. One of the advantages of the formulation presented here, based on the spectral element method (SEM), is its accuracy to provide solutions in the high frequency range. Therefore, this approach in conjunction with the use of PZT actuator-sensors mounted on the structure might be used for a more reliable debonding detection between an FRP strip and the host RC structure. The updating procedure is solved by using an ensemble particle swarm optimization (PSO)-based approach with a bagging algorithm. This new proposal also represents another contribution of this paper which yields more accurate and reliable predictions in complex structural identification problems, such as ours, than using a single PSO. Ensemble learning is a tool by which multiple decision-makers, such as classifiers or learning machines, are combined to make the final decision based on the individual decisions of each decision-maker.

Finally, with the purpose of improving the robustness of the method to detect damage location, another algorithm, an adaptive algorithm of spectral element mesh, has also been developed in this work and validated with experimental results.

\section{Spectral element model for bonded-PZT FRP strengthened beam}

Fig. 1 shows a typical example of an FRP strengthened concrete beam with one PZT sensor-actuator bonded. It consists of a PZT layer, an adhesive layer, an FRP layer and the base concrete beam. A spectral element approach was implemented in $[19,20,27]$, and in the presented study, a one-dimensional spectral model will be developed to simulate the behavior of FRP flexural strengthened beams instrumented with PZT impedance sensors bonded regularly along the FRP strip. The strengthened beam will be composed of three different types of zones depending on the materials: (a) Only concrete beam; (b) concrete beam with FRP bonded; (c) FRP strengthened RC beam bonded with a PZT patch. Therefore, the numerical model will consist of spectral concrete beam elements, FRP plus concrete elements and bonded-PZT FRP plus concrete elements. The formulation of the first two types was developed in [28]. The formulation of bonded-PZT FRP strengthened concrete elements will be carried out in this study.

\subsection{Spectral PZT-bonded FRP strengthened concrete element}

In the FRP strengthened concrete beam shown in Fig. 1, the geometrical parameters $l_{C}, l_{F R P}$ and $l_{P Z T}$ denote the length of

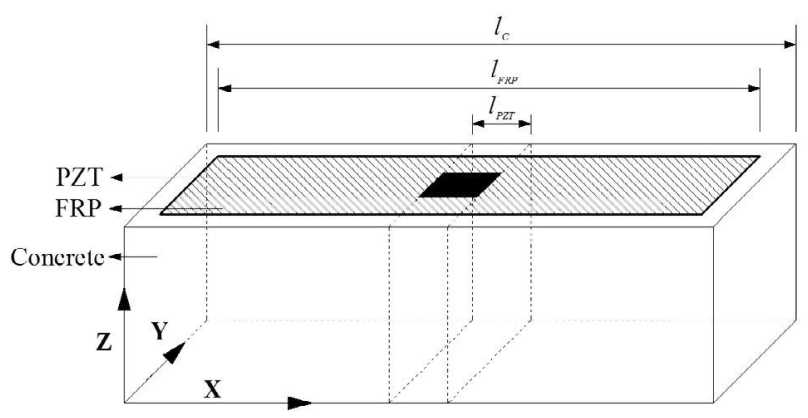

Fig. 1. Geometry of FRP strengthened concrete beam with a PZT sensor-actuator. 
concrete beam, FRP strip and piezoelectric sensor, respectively. Since the spectral method treats the mass distribution exactly, if no damage occurs only five elements would be needed initially to simulate a beam such as that shown in Fig. 1, two for the end portions of concrete without FRP, one for the portion of the beam in which the PZT sensor is bonded and another two for the FRPconcrete regions without PZT. The Cartesian coordinate system $x-y-z$ corresponds to the direction of length, width and height of the beam. The cross section of an element with PZT bonded is shown in Fig. 2, where $b_{C}, b_{F R P}$ and $b_{P Z T}$ represent the width of the concrete beam, FRP and PZT layer, respectively, and, similarly $e_{C}, e_{A D}, e_{F R P}$ and $e_{P Z T}$ are the thicknesses of concrete beam, adhesive layer, FRP and PZT sensor, respectively.

In order to simplify the physical model, several assumptions were made in order to derive the governing equations for the electromechanical coupled system.

1. The geometric and material characteristics are uniform along the length of the beam.

2. The slip between FRP and concrete beam is considered.

3. Perfect bonding is assumed for PZT, which means the slip at the FRP-PZT interface is neglected. Although the adhesive layer between PZT and the host beam might affect the measurement of electrical impedance, Yan's work [16] demonstrated that no peak shift occurred at the conductance (real part of the inverse of the impedance) with the growth of the viscosity of adhesive, and the resonant peaks experimented a slight shift when the thickness of adhesive increased.

4. The coupled system has the same transverse displacement.

5. The voltage is uniformly applied on the PZT.

6. The force generated by piezoelectric effect is distributed uniformly along the cross section area of PZT.

7. Due to small amplitude vibration, linear elasticity and piezoelectricity are feasible in this case.

Most of the above assumptions were developed by Lee [22], and considering the characteristics of the proposed model, some other

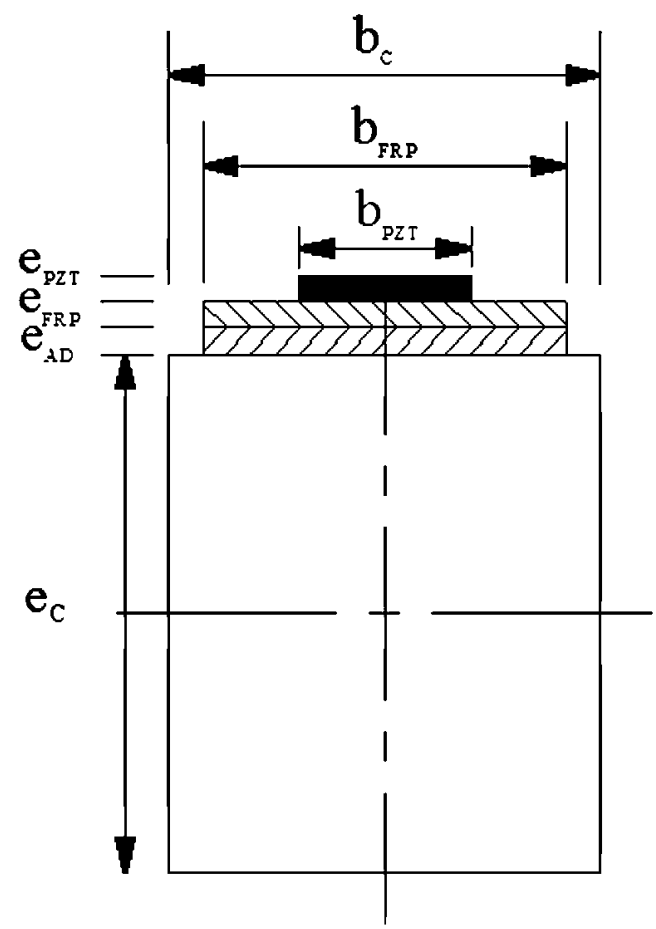

Fig. 2. Cross section of FRP strengthened concrete beam with a PZT sensoractuator. points are added in this paper. Fig. 3 defines the kinematics of the bonded-PZT FRP strengthened beam, where $u_{0}, w, \phi$ and $s$ are the mid-plane axial displacement, the transverse displacement, the rotation of the beam cross-section about the $Y$-axis and the slip of the FRP-concrete interface, respectively. The transverse displacement is constant for all the cross-section, i.e $w(x, z, t)=w(x, t)$, where $t$ is the time.

Considering perfect bonding at the PZT-FRP interface we have the following kinematic relationships

$u_{C}(x, z, t)=u_{0}(x, t)-z \phi(x, t)$

$u_{\mathrm{FRP}}(x, z, t)=u_{0}(x, t)-z \phi(x, t)+s(x, t)$

$u_{P Z T}(x, z, t)=u_{0}(x, t)-z_{P Z T} \phi(x, t)+s(x, t)$

where the subscripts $C$, FRP and PZT represent the concrete base beam, the FRP strip and the PZT sensor, respectively; $z_{P Z T}$ is the PZT coordinate measured from the mid-plane and is given by

$z_{P Z T}=\frac{e_{C}}{2}+e_{F R P}+\frac{e_{P Z T}}{2}$

Thus the linear strain of the PZT sensor takes the following form

$\varepsilon_{x P Z T}=u_{0, x}-z_{P Z T} \phi_{x}+s_{, x}$

where the subscript ()$_{x}$ denotes the differentiation with respect to $x$.

It is assumed that the PZT actuator-sensor has only $h_{31}$ effect, i.e., the induced stress is only in the $1-1(x)$ direction when an electrical voltage is applied in the $3-3(z)$ direction. The constitutive equations of PZT are given by

$\left\{\begin{array}{l}\sigma_{X P Z T}=\bar{Y}_{11} \varepsilon_{X P Z T}-h_{31} D \\ E=-h_{31} \varepsilon_{X P Z T}+\beta_{33} D\end{array}\right.$

where $\sigma_{X P Z T}$ is the axial stress of PZT in the $x$-direction, respectively. represents the electrical displacement and is the electrical field in the $z$-direction. $\bar{Y}_{11}^{E}=Y_{11}^{E}(1+\eta i)$ is the complex Young's modulus of the PZT in the $1-1(x)$ direction, where $\eta$ denotes the mechanical loss factor. $h_{31}$ is the piezoelectric constant, and $\beta_{33}$ is the dielectric constant in the 3-3 $(z)$ direction.

If a voltage $V(t)$ is applied to the PZT layer in a perpendicular direction, the electrical field is as follows

$E=\frac{V(t)}{e_{P Z T}}$

By replacing Eq. (7) in Eq. (6), the electrical displacement and mechanical stress can be written as

$D=\frac{V(t)}{\beta_{33} e_{P Z T}}+\frac{h_{31}}{\beta_{33}} \varepsilon_{x P Z T}$

$\sigma_{X P Z T}=E_{P} \varepsilon_{x P Z T}-\frac{h_{31}}{\beta_{33} e_{P Z T}} V(t)$

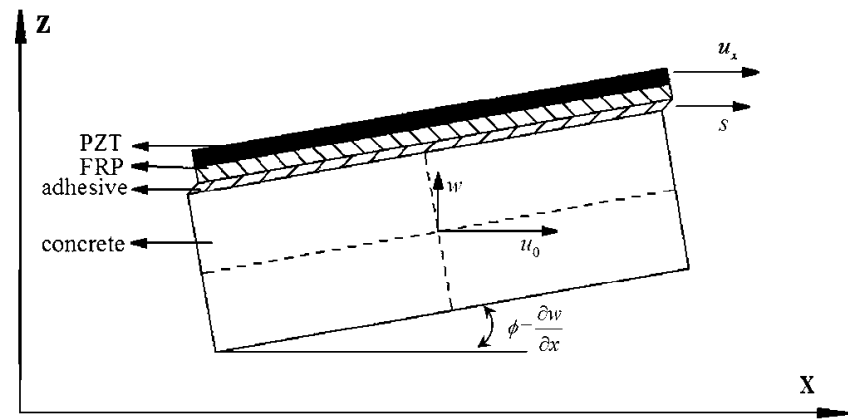

Fig. 3. Deformation of a bonded-PZT FRP strengthened RC beam. 
where

$E_{P}=\bar{Y}_{11}-\frac{h_{31}^{2}}{\beta_{33}}$

The equations of motion are derived by using Hamilton's principle which requires the evaluation of the strain energy $U$, the kinetic energy $T$ and the virtual work $\delta W$. The strain energy of the coupled system PZT + FRP + concrete is obtained by adding the contribution to the strain energy of the concrete section strengthened by externally bonded FRP strip, evaluated in [28], and the contribution of the PZT sensor, which can be computed as follows

$U_{P Z T}=\frac{1}{2} \int_{I_{P Z T}} \int_{z_{P Z T 1}}^{z_{P Z T 2}}\left(\sigma_{x P Z T} \varepsilon_{x P Z T}+E D\right) b_{P Z T} d z d x$

where $z_{P Z T 1}$ and $z_{P Z T 2}$ represent the $Z$-coordinates of bottom and top surfaces limiting the PZT sensor. Since a perfect bonding condition has been assumed at the PZT-FRP interface, the contribution to the strain energy of this interface is neglected.

On the other hand, compared to FRP and concrete beam, the contribution of PZT to the kinematic energy might be neglected; it would make the derivation of the governing equations more convenient without losing fidelity. Then the kinematic energy of the FRP + concrete system is given by [28]

$T=\frac{1}{2} \int_{V}\left\{\rho_{C}\left[\dot{u}_{C}^{2}+\dot{w}^{2}\right]+\rho_{F R P}\left[\dot{u}_{F R P}^{2}+\dot{w}^{2}\right]\right\} d V$

Finally, to the virtual work carried out in the concrete and FRP sections [28], we should add the virtual work in PZT sensor obtaining the following expression

$$
\begin{aligned}
\delta W_{P Z T}= & \int_{0}^{l_{P Z T}}\left[b_{P Z T} V(t) \delta D\right] d x+\bar{N}_{0} \delta u_{0}(0)+\bar{N}_{l_{P Z T}} \delta u_{0}\left(l_{P Z T}\right) \\
& +\bar{Q}_{0} \delta w(0)+\bar{Q}_{I_{P Z T}} \delta w\left(l_{P Z T}\right)+\bar{M}_{0} \delta \phi(0)+\bar{M}_{l_{P Z T}} \delta \phi\left(l_{P Z T}\right) \\
& +\overline{N_{0}^{*}} \delta s(0)+\bar{N}_{l_{P Z T}}^{*} \delta s\left(l_{P Z T}\right)
\end{aligned}
$$

where $\bar{N}, \bar{Q}, \bar{M}$ and $\overline{N *}$ represent the boundary forces and moments associated with the kinematic variables $u_{0}, w, \phi$ and $s$,respectively. Subscript 0 and $l_{P Z T}$ are referred to the ends of the PZT sensor.

Replacing Eqs. (1), (2), (5), (8), (9) and (10) in Eqs. (12)-(14) considering also the FRP + RC contributions and applying Hamilton's principle, the governing equations of the coupled electro-mechanical system take the following form

$$
\begin{aligned}
\delta u_{0}: & I_{0} \ddot{u}_{0}-I_{1} \ddot{\phi}+I_{0 F R P} \ddot{s}-A_{11} u_{0, x x}+B_{11} \phi_{, x x}-A_{F R P} S_{, x x}=0 \\
\delta w: & I_{0} \ddot{w}-A_{22} w_{, x x}+A_{22} \phi_{, x}=0 \\
\delta \phi: & I_{2} \ddot{\phi}-I_{1} \ddot{u}_{0}-I_{1 F R P} \ddot{s}+B_{11} u_{0, x x}-D_{11} \phi_{, x x}-A_{22} w_{, x} \\
& +A_{22} \phi+B_{F R P} S_{x x}=0 \\
\delta s: & I_{0 F R P} \ddot{s}+I_{0 F R P} u_{0}-I_{1 F R P} \ddot{\phi}-A_{F R P} S_{, x x}-A_{F R P} u_{0, x x} \\
& +B_{F R P} \phi_{, x x}+\frac{G_{A D} s b_{A D}}{e_{A D}}=0
\end{aligned}
$$

where $(\bullet)$ represents temporal derivative and ( $)_{, x x}$ denotes double differentiation with respect to $x$. The coefficients associated with materials properties are given as

$$
\begin{aligned}
& {\left[\begin{array}{lll}
A_{11} & B_{11} & D_{11}
\end{array}\right]=\int_{z_{P Z 1}}^{z_{P Z T 2}} E_{P}\left[1 \quad z \quad z^{2}\right] b_{P Z T} d z+\int_{z_{C 1}}^{z_{C 2}} E_{C}\left[\begin{array}{lll}
1 & z & z^{2}
\end{array}\right] b_{C} d z} \\
& +\int_{z_{F R P 1}}^{z_{F R P 2}} E_{F R P}\left[1 \quad z \quad z^{2}\right] b_{F R P} d z \\
& {\left[A_{22}\right]=\int_{z_{C 1}}^{z_{C 2}} G_{C} b_{C} d z+\int_{z_{F R P 1}}^{z_{F R P 2}} G_{F R P} b_{F R P} d z} \\
& {\left[\begin{array}{ll}
A_{F R P} & B_{F R P}
\end{array}\right]=\int_{z_{P T 11}}^{z_{P Z T 2}} E_{P}\left[\begin{array}{ll}
1 & z
\end{array}\right] b_{P T T} d z+\int_{z_{F R P 1}}^{z_{F R P 2}} E_{F R P}\left[\begin{array}{ll}
1 & z
\end{array}\right] b_{P Z T} d z}
\end{aligned}
$$

where $z_{\mathrm{C} 1}, z_{\mathrm{C} 2}$ and $z_{\mathrm{FRP} 1}, z_{\mathrm{FRP} 2}$ represent the $z$-coordinates of bottom and top surfaces for concrete and FRP respectively., are the elastic modulus and elastic shear modulus of each material ( $C$ : concrete, FRP: FRP plate, AD: Adhesive). It can be noticed that the contributions of the coefficients presented above are not only from material properties but also the electro-mechanical coupling via the connection between the PZT and host structure, which makes sense in the proposed model. In addition, the coefficients related to inertial terms are defined as

$\left[\begin{array}{lll}I_{0} & I_{1} & I_{2}\end{array}\right]=\int_{z_{C 1}}^{z_{C 2}} \rho_{C}\left[\begin{array}{lll}1 & z & z^{2}\end{array}\right] b_{C} d z+\int_{z_{F R P 1}}^{z_{F R P 2}} \rho_{\mathrm{FRP}}\left[\begin{array}{lll}1 & z & z^{2}\end{array}\right] b_{\mathrm{FRP}} d z$

$\left[\begin{array}{ll}I_{0 F R P} & I_{1 F R P}\end{array}\right]=\int_{z_{F R P}}^{z_{F R P 2}} \rho_{F R P}[1 \quad z] b_{F R P} d z$

The boundary conditions are also obtained from Hamilton's principle as (Fig. 4)

$$
\begin{array}{rll}
N(0, t)=-\bar{N}(0, t)+N^{p}(t) & \text { or } & u_{0}(0, t)=\overline{u_{0}}(0, t) \\
Q(0, t)=-\bar{Q}(0, t) & \text { or } & w(0, t)=\bar{w}(0, t) \\
M(0, t)=-\bar{M}(0, t)-M^{p}(t) & \text { or } & \phi(0, t)=\bar{\phi}(0, t) \\
N^{*}(0, t)=-\overline{N^{*}}(0, t) & \text { or } & s(0, t)=\bar{s}(0, t) \\
N\left(l_{P Z T}, t\right)=\bar{N}\left(l_{P Z T}, t\right)+N^{p}(t) & \text { or } & u_{0}\left(l_{P Z T}, t\right)=\overline{u_{0}}\left(l_{P Z T}, t\right) \\
Q\left(l_{P Z T}, t\right)=\bar{Q}\left(l_{P Z T}, t\right) & \text { or } & w\left(l_{P Z T}, t\right)=\bar{w}\left(l_{P Z T}, t\right) \\
M\left(l_{P Z T}, t\right)=\bar{M}\left(l_{P Z T}, t\right)-M^{p}(t) & \text { or } & \phi\left(l_{P Z T}, t\right)=\bar{\phi}\left(l_{P Z T}, t\right) \\
N^{*}\left(l_{P Z T}, t\right)=\overline{N^{*}}\left(l_{P Z T}, t\right) & \text { or } & s\left(l_{P Z T}, t\right)=\bar{s}\left(l_{P Z T}, t\right)
\end{array}
$$

where $\overline{u_{0}}, \bar{w}, \bar{\phi}$ and $\bar{s}$ are the kinematic variables specified at the boundaries and $N^{p}(t)$ and $M^{p}(t)$ are the piezoelectric forces and moments generated by the driving voltage $V(t)$ applied to the PZT layer. The stress resultants $N, Q, M$ and $N^{*}$ are defined in terms of the displacement field as follows

$N=A_{11} u_{0, x}-B_{11} \phi_{, x}+A_{F R P} S_{, x}$

$Q=A_{22} w_{, x}-A_{22} \phi$

$M=-B_{11} u_{0, x}+D_{11} \phi_{, X}-B_{F R P} S_{, X}$

$N^{*}=A_{F R P} S_{, X}+A_{F R P} u_{0, x}-B_{F R P} \phi_{, X}$

The governing Eqs. (15)-(18) and the boundary conditions (24)(28) are taken as starting point to apply SEM. In SEM, the unknown displacement field, $\{u\}=\left\{u_{0}(x, t), w(x, t), \phi(x, t), s(x, t)\right\}, \quad$ is approximated as

$\{u\}=\sum_{n=1}^{N}\left\{\hat{u}\left(x, \omega_{n}\right)\right\} e^{-j \omega_{n} t}=\sum_{n=1}^{N}\left(\sum_{m=1}^{M}\left\{\hat{u}^{*}\right\}_{m n} e^{-j k_{m n} x}\right) e^{-j \omega_{n} t}$

where $\omega_{n}$ denotes the $n$th circular frequency, $N$ is the number of frequency points while implementing the Fast Fourier Transform (FFT) algorithm and $\hat{u}\left(x, \omega_{n}\right)$ represents the spectral amplitude vector corresponding to the generic displacement vector as a function of $\left(x, \omega_{n}\right)$. A Fourier expansion of $\hat{u}\left(x, \omega_{n}\right)$ has also been carried out in the longitudinal direction where $\left\{\hat{u}^{*}\right\}_{m n}=\left(\hat{u}_{0}, \hat{w}, \hat{\phi}, \hat{s}\right)_{m n}$ represents the wave coefficient vector associated with the $m$ th mode of wave and for each frequency and. $k_{m n}$ denotes the $m$ th wave number related to $n$th frequency $\omega_{n}$. Replacing (29) in (15)-(18), the following Polynomial Eigenvalue Problem is obtained:

$[W(k)]\left\{\hat{u}^{*}\right\}=0$

where $W$ is the matrix polynomial depending on the wavenumbers $k_{m n}$ for each frequency $\omega_{n}$. In this case, the order of the matrix 


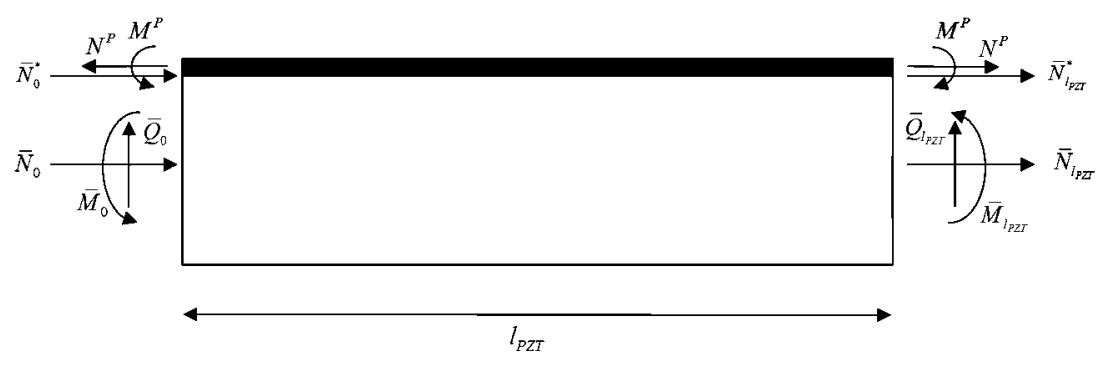

Fig. 4. External forces of an FRP-PZT element.

polynomial is 8 . Thus, there are eight eigenvalues $\left(k_{m n}\right.$ where $m=1, \ldots, 8)$ and eigenvectors $\left(\left\{\hat{u}^{+}\right\}\right)$. After solving the eigenvalue problem, the complete solution at frequency $\omega_{n}$ is written as

$$
\begin{aligned}
\left\{\hat{u}\left(x, \omega_{n}\right)\right\} & =\left\{\begin{array}{l}
\hat{u}_{0}\left(x, \omega_{n}\right) \\
\hat{w}\left(x, \omega_{n}\right) \\
\hat{\phi}\left(x, \omega_{n}\right) \\
\hat{s}\left(x, \omega_{n}\right)
\end{array}\right\}=\left[\bar{R}_{1} \bar{R}_{2} \ldots \bar{R}_{8}\right] \underset{m=1, \ldots 8}{\operatorname{diag}\left(e^{-j k_{n n} x}\right)}\left\{\begin{array}{c}
a_{1} \\
a_{2} \\
\ldots \\
a_{8}
\end{array}\right\} \\
& =[R][D(x)]\left\{A\left(\omega_{n}\right)\right\}
\end{aligned}
$$

where $\bar{R}_{i}$ denotes the normalized eigenvector related to $k_{1 n}$ and $\left\{A\left(\omega_{n}\right)\right\}$ is a vector of unknown coefficients to be determined from the displacement boundary conditions at $x=0$ and $x=\mathrm{L}$

$$
\left(\begin{array}{l}
\left\{\hat{\boldsymbol{u}}_{1}\right\}_{n} \\
\left\{\hat{\boldsymbol{u}}_{2}\right\}_{n}
\end{array}\right)=\left[\begin{array}{l}
R \\
R
\end{array}\right]\left[\begin{array}{c}
D(0) \\
D(L)
\end{array}\right]_{n}\{A\}=\left[T_{1}\right]\{A\}
$$

Similarly the associated forces are evaluated at the nodes according to Eqs. (25)-(28) as

$$
\left(\begin{array}{c}
\left\{f_{1}\right\} \\
\left\{f_{2}\right\}
\end{array}\right)=\left[T_{2}\right]\{A\}=\left[T_{2}\right]\left[T_{1}\right]^{-1}\left(\begin{array}{c}
\left\{\hat{u}_{1}\right\}_{n} \\
\left\{\hat{u}_{2}\right\}_{n}
\end{array}\right)=[K]\left(\begin{array}{c}
\left\{\hat{u}_{1}\right\}_{n} \\
\left\{\hat{u}_{2}\right\}_{n}
\end{array}\right)
$$

Once $\left[T_{1}\right]$ and $\left[T_{2}\right]$ are obtained, the dynamic stiffness matrix for the spectral PZT-bonded FRP strengthened concrete element is formed as $\left[T_{2}\right]\left[T_{1}\right]^{-1}$.

\subsection{Piezoelectric spectral nodal forces}

As commented above the electric field is generated by a voltage $V(t)$ applied to the PZT layer in perpendicular direction, which should also be represented in spectral form

$V(t)=\sum_{n=1}^{N} V\left(\omega_{n}\right) e^{-j \omega_{n} t}$

The driving voltage applied to the PZT layer as actuator will generate a piezoelectric force $N^{p}$ and a moment $M^{p}$. These actions will contribute to the spectral nodal forces in Eq. (33) as a consequence of the electro-mechanical coupling at the PZT actuator. Their expression is obtained by integrating the virtual work due to the PZT layer (Eq. (14)) and is given in spectral form as follows

$N^{p}(t)=\sum_{n=1}^{N} N^{p}\left(\omega_{n}\right) e^{-j w_{n} t}=\sum_{n=1}^{N} E_{p} b_{P Z T} d_{31} V\left(\omega_{n}\right) e^{-j w_{n} t}$

$M^{p}(t)=\sum_{n=1}^{N} M^{p}\left(\omega_{n}\right) e^{-j w_{n} t}=\sum_{n=1}^{N} E_{p} b_{P Z T} Z_{P Z T} d_{31} V\left(\omega_{n}\right) e^{-j w_{n} t}$

where $d_{31}$ is the piezoelectric strain constant defined by

$d_{31}=\frac{h_{31}}{E_{p} \beta_{33}}$

\section{Electro-mechanical impedance}

In the EMI method, PZT transducers are bonded through surface by means of high strength epoxy adhesive to the structure to be monitored. The principle behind this technique is to apply highfrequency structural excitations through PZT transducers, and measure the impedance of structures. The electromechanical admittance (inverse of the impedance) signatures of the PZT acquired at different times are used to evaluate the damage since these are related to the mechanical impedance of the host beam. Hence, the measurement of impedance can reflect the changes of material properties which might be due to damages.

The one-dimensional impedance model for the PZT-structure interaction system derived by Liang et al. [29] for a beam is adopted in this study to closely simulate the real situation, where the PZT transducer is assumed to interact with the host structure.

$$
\begin{aligned}
Y(\omega) & =G(\omega)+j B(\omega) \\
& =j \omega \frac{b_{P Z T} l_{P Z T}}{e_{P Z T}}\left(\bar{\varepsilon}_{33}^{T}-\frac{Z_{s}(\omega)}{Z_{s}(\omega)+Z_{a}(\omega)} d_{31}^{2} \bar{Y}_{11}^{E}\right)
\end{aligned}
$$

where $Z_{a}(\omega)$ and $Z_{s}(\omega)$ are the mechanical impedances of the PZT and the host structure, respectively, $\omega$ is the angular frequency of the driving voltage, $d_{31}$ is the piezoelectric strain constant between $z$ and $x$ directions at zero stress, $j$ is the imaginary unit and the dimensions of the PZT sensor were defined in the previous section. In addition, $\bar{\varepsilon}_{33}^{T}=\varepsilon_{33}^{T}(1-\delta i)$ is the complex electric permittivity of the PZT at constant stress, where $\delta$ denotes the dielectric loss factor. $\bar{Y}_{11}^{E}$ was defined in the previous section. The electromechanical admittance signatures Yconsists of a real part (the conductance), denoted by $G$ in Eq. (38) and an imaginary part (the susceptance), denoted by $B$ in Eq. (38). Conductance has been traditionally used for structural health monitoring due to its better indication of structural changes.

Eq. (38) gives the frequency response of the electrical admittance. The first term on the right side is only related to PZT properties, while the second term reflects the electromechanical coupling between the host structure and PZT sensor. Furthermore, the resonance condition of the FRP-strengthened concrete beam bonded with a PZT patch coupled system is also indicated in the second term. The mechanical impedance $Z_{a}(\omega)$ of the PZT transducer is computed as follows [29]

$Z_{a}(\omega)=-j \frac{\kappa \bar{Y}_{11}^{\mathrm{E}} b_{P Z T} e_{P Z T}}{\omega \tan \left(\kappa l_{P Z T}\right)}$

where $\kappa$ is the wave number, related to the angular frequency of excitation by $\kappa=\omega \sqrt{\rho / Y_{11}^{E}}$, where $\rho$ is the density of the PZT. The mechanical structural impedance $Z_{s}(\omega)$, defined as the ratio of the excitation force to the velocity response, may be expressed as

$Z_{s}(\omega)=-j \frac{E_{p} b_{P Z T} d_{31} V(\omega)}{\omega u_{s}(\omega)}$ 
where the axial displacement $u_{s}(\omega)$ of the structure in frequency domain can be obtained by implementing the spectral element approach presented in the previous section. Therefore, combining Eqs. (38)-(40) and the spectral model proposed in the previous section, the numerical electro-mechanical impedance can be easily calculated. In this way, a simple model of the numerical impedance applicable for structural health monitoring applications using the spectral element method to enable high frequency analysis is developed.

\section{Damage identification by using ensemble particle swarm optimization (EPSO)}

\subsection{Damage identification methodology}

The conventional EMI method assesses the integrity of a mechanical structure by observing some of the changes in the electromechanical impedance of the PZT between two different stages. For this, different scalar damage metrics are defined which allow for a quantitative measurement of the damage severity. In this sense, the root mean square deviation (RMSD) and cross-correlation coefficient (CC) are the most commonly used indicators for the impedance method [7]. They are computed from the difference in the impedance value at each frequency as follows

$\operatorname{RMSD}(\%)=\sqrt{\frac{\sum_{i=1}^{n}\left\{Z_{1}\left(\omega_{i}\right)-Z_{0}\left(\omega_{i}\right)\right\}^{2}}{\sum_{i=1}^{n}\left\{Z_{0}\left(\omega_{i}\right)\right\}^{2}}} \times 100$

$\mathrm{CC}=\frac{1}{n} \sum_{i=1}^{n} \frac{\left\{Z_{0}\left(\omega_{i}\right)-\bar{Z}_{0}\right\}\left\{Z_{1}\left(\omega_{i}\right)-\bar{Z}_{1}\right\}}{\sigma_{Z_{0}} \sigma_{Z_{1}}}$

where $Z_{0}\left(\omega_{i}\right)$ is the impedance of the PZT measured at a previous stage, which might agree with the healthy condition of the structure, and $Z_{1}\left(\omega_{i}\right)$ is the corresponding value at a subsequent stage, which might agree with a post-damage stage, at the ith frequency point; $n$ is the number of frequency points; $\bar{Z}_{0}\left(\omega_{i}\right)$ and $\bar{Z}_{1}\left(\omega_{i}\right)$ are the mean values of the two data sets, $Z_{0}\left(\omega_{i}\right)$ and $Z_{1}\left(\omega_{i}\right)$, respectively, and $\sigma_{Z_{0}}$ and $\sigma_{Z_{1}}$ are the standard deviations of the two corresponding data sets, $Z_{0}\left(\omega_{i}\right)$ and $Z_{1}\left(\omega_{i}\right)$. Considering that the real part of impedance is more sensitive to the changes in impedance signature, Eqs. (41) and (42) are also usually defined in terms of the conductances. For RMSD index, the larger the difference between the baseline reading and the subsequent reading, the greater the value of the index, while for $\mathrm{CC}$ index the difference between the baseline reading and the subsequent reading increases with the decreasing value of the $\mathrm{CC}$ metric. For this reason, it is common to use " 1 -CC" instead of CC to determine the increase in damage index by increasing the severity of damage. In any case, both situations denote changes of structural dynamic properties which can be due to damage.

However, although the conventional EMI method has been successfully applied for various engineering structures for damage detection $[12,30,31]$, it predicts only the existence or not of structural damage but does not give any information about the variation of the structural properties or a direct identification of damage location. To solve this, a model updating procedure is proposed. It is based on the spectral element model developed in Section 2 which is correlated to the electrical impedance response of the PZT sensors bonded to different points of the structure. The electrical admittance signatures are assigned to the spectral element model of the pre-damaged structure in such a way that the numerically computed electrical impedance change matches the measured counterpart as closely as possible through an optimization algorithm. The spectral element model of the pre-damaged state is then updated to represent the real damaged structure and allows, therefore, identifying the damage location and its extent. To reach this purpose, one or several objective functions able to detect the deviation between the impedance response of the numerical model and that measured from the experimental test should be defined. The basis of the damage identification procedure is to adjust the damage parameters of the numerical model until reaching a minimum of the objective functions by using an optimization technique.

Therefore, two points should be initially solved to implement the procedure, the definition of the objective functions and the choice of the updating parameters to associate the damage state with the change of structural properties. The choice of both depends mainly on the characteristics of the physical problem to be solved.

For FRP strengthened RC structures, FRP intermediate debonding is a common kind of damage, which is probably caused by flexural intermediate crack in the RC structure. After being activated by high shear stress transmitted from an adhesive interface near flexural cracks, this kind of damage propagates towards the end of the FRP strip, which usually leads to a brittle form of structural failure. However, this failure mode in its initial stages is very difficult to identify due to its local nature. Only the use of high frequency measurements such as those due to the electrical impedance would make it possible to detect minor debonding. Since PZT properties remain constant, any change in the electrical impedance signature is an indication of a change in the structural integrity which may be caused by the presence of structural damage. Hence, the sensitivity of this technique gives us a powerful approach to capture the incipient changes which are probably caused by minor defects and flaws in the structure, such as those originating FRP debonding failure. Therefore, the objective functions should be formulated using impedances. Taking Eq. (42) as starting point an objective function to evaluate the agreement between experimental and numerical impedances for each sensor $k$ can be defined by using the modal assurance criterion (MAC) [32] as follows

$$
\begin{aligned}
F_{k} & =1-\operatorname{MAC}\left(\{1-\mathrm{CC}\}_{\text {num }},\{1-\mathrm{CC}\}_{\text {exp }}\right) \\
& =1-\frac{\left(\{1-\mathrm{CC}\}_{\text {num }}^{\mathrm{T}}\{1-\mathrm{CC}\}_{\text {exp }}\right)^{2}}{\left(\{1-\mathrm{CC}\}_{\text {num }}^{\mathrm{T}}\{1-\mathrm{CC}\}_{\text {num }}\right)\left(\{1-\mathrm{CC}\}_{\text {exp }}^{\mathrm{T}}\{1-\mathrm{CC}\}_{\text {exp }}\right)}
\end{aligned}
$$

where the subscripts num and exp are used for the computed and measured responses, respectively. This function, applied to each $k$ th sensor, has the advantage that its value is between 0 and 1 . An index equal to one means no correlation between the sets of experimental and numerical impedances, while a value equal to zero indicates a perfect correlation between the updated numerical model and the experimental results. Furthermore, in Eq. (43), a CC indicator will be applied by using only the real part of the electrical impedance due to its higher sensitivity to structural damage.

Usually impedance signals are constructed over a wide frequency range including thousands of sampling points and the indicators in Eqs. (41) and (42) are applied for the entire frequency range. However, it has been demonstrated [30] that the damage identification is more reliable and robust if RMSD and CC are calculated by using different sub-ranges of frequency since there is a relationship between the frequency range and the PZT sensing region. Because of this, the objective function in Eq. (43) is defined by using vectors of the form $\{1-\mathrm{CC}\}$ whose terms are the result of applying Eq. (42) to multiple sub-ranges of frequency into which the whole signal is split.

One advantage of using an objective function based on $\mathrm{CC}$ coefficient is due to the fact that this indicator is defined both numerically and experimentally between two subsequent stages. Therefore, any modeling error in a previous structural stage will also be present in the subsequent stage and, therefore, will be 
compensated in large part [33]. In this way, it might be avoided that inherent errors in the model produce changes greater than those produced by the damage itself.

The other keypoint to be solved regards the choice of the updating parameters to associate the damage state with the change of structural properties. The local damage identification within the strengthened beam requires the division of the beam into a number of spectral elements. The change of structural properties of each element $e$ should be related to the phenomenon to be modeled, in this case, minor debonding at the concrete-FRP interface. Because of this, a damage parameter $d_{e}$ might be defined for each element $e$ which would denote the extent of interfacial damage, i.e. the stiffness change at the RC-FRP interface. It can be expressed as

$d_{e}=1-\frac{\left(G_{A D}\right)_{d}^{e}}{\left(G_{A D}\right)^{e}}$

where $\left(G_{A D}\right)^{e}$ and $\left(G_{A D}\right)_{d}^{e}$ are the adhesive stiffnesses for element $e$ before and after debonding damage, respectively. The case of $d_{e}=0$ indicates the intact state while $d_{e}=1$ means that the element $e$ has debonded completely.

\subsection{Ensemble particle swarm optimization (EPSO)}

The unknown damage parameters defined in Eq. (44) are derived by solving an optimization procedure able to minimize the objective functions which fit the numerical structural impedance with that extracted from the experimentally measured PZT sensors. The problem will be single or multi-objective depending on if one or several sensors are used, respectively.

In recent years evolutionary algorithms have been frequently used with promising results in systems identification $[34,35]$. Additionally, evolutionary algorithms are especially effective to solve multi-objective optimization problems when compared to traditional optimization algorithms since all Pareto points are obtained in only one run without any necessity of combining a priori the objective functions using arbitrary weighting factors. These algorithms are largely-population based and have a similar architecture. From a population of individuals randomly initiated the suitable solutions are searched through the interaction among the different individuals by using an iterative procedure. The differences among the algorithms are mainly due to how the population of individuals is updated and how the individuals interact with one another. Different optimization techniques such as Genetic Algorithm (GA), Simulated Annealing (SA) and Particle Swarm Optimization (PSO) have been used for FEM updating $[36,37]$.

In this paper we have selected PSO due to its simplicity, fast convergence and the few parameters to be adjusted, which make it a high competitor in solving single and multi-objective problems when compared to other methods. PSO was introduced by Kennedy and Eberhart [38] and is based on the simulation of the social behavior of bird flocking or fish schooling. Each particle or individual within the swarm is initialized and then is assumed to fly over the search space looking for promising territory which possesses best fitness of the objective function by updating its position and velocity. The updated velocity $v_{i}^{k+1}$ and position $x_{i}^{k+1}$ for particle $i$ are formulated as follows

$v_{i}^{k+1}=w \cdot v_{i}^{k}+c_{1} \cdot r_{1} \cdot\left(\right.$ pbest $\left._{i}-x_{i}^{k}\right)+c_{2} \cdot r_{2} \cdot\left(\right.$ gbest $\left.-x_{i}^{k}\right)$

$x_{i}^{k+1}=x_{i}^{k}+v_{i}^{k+1}$

where pbest $_{i}$ is the best previous position and gbest is the best position shared by all other particles; $c_{1}$ and $c_{2}$ are acceleration constants which control the influence of pbest $t_{i}$ and gbest on each search step, and usually their values are suggested to be 2 for the sake of convergence [39]; $r_{1}$ and $r_{2}$ are two random numbers in the range $[0,1]$ to prevent convergence on local optimal locations. Furthermore, $w$ denotes an inertia weight to control the impact of the previous particle velocity on the current particle velocity, and is given by

$w=w_{\max }-\frac{w_{\max }-w_{\min }}{\text { iter }_{\max }} \cdot$ iter

where $w_{\max }$ and $w_{\min }$ are the initial and final weight respectively. iter $_{\max }$ is the maximum iteration number, and iter is the current iteration number.

Debonding damage identification in its previous stages by using electromechanical impedance represents a challenging task considering that we want to detect minor debonding and that the impedance changes may be caused not only by the presence of structural damage but also by the variations in the geometrical conditions, the environmental temperature and the noise. In this sense with the purpose of improving the performance and accuracy of the predictions an ensemble PSO algorithm with a bagging (bootstrap aggregating) algorithm $[40,41]$ is proposed. Ensemble methods are implemented as a combination of predictions made by a set of individual classifiers or machine learning algorithms, in this case, based on PSO (Fig. 5). Ensemble learning has proven to be very effective and versatile in real-world applications [42]. Its success is mainly based on ensemble-based decision making, which is usually realized by a voting classification approach, such as a bagging algorithm. By using this algorithm the identification problem is run multiple times by changing the distribution of training set instances. Each training set is called a bootstrap sample and for our particular problem represents a random set of individuals or particles in the search space. Then, a different classifier or sub-classifier is obtained by applying PSO algorithm to each of the bootstrap samples. A final classifier, the final damage identification, is constructed from the previous sub-classifiers whose output is the class predicted most often by the sub-classifiers. By using this approach the accuracy of the identification procedure will increase.

\section{Experimental test}

In order to validate the feasibility of the proposed spectral approach and check the ability of the proposed model updating method as a damage identification procedure, a series of experimental tests were performed on a concrete block strengthened with an FRP strip as illustrated in Fig. 6. The dimensions of the specimen are $31.3 \mathrm{~cm}$ long, $9.5 \mathrm{~cm}$ wide and $7.5 \mathrm{~cm}$ deep and

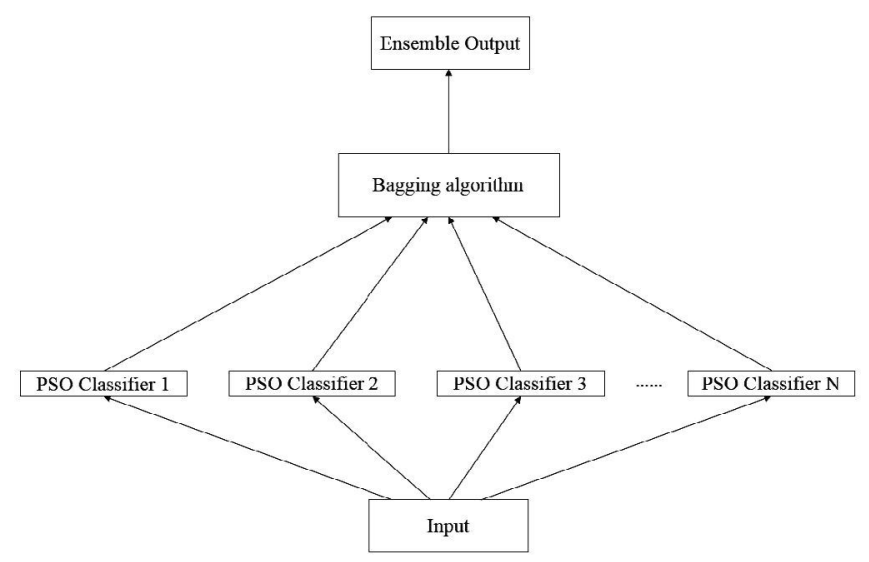

Fig. 5. Proposed ensemble method. 


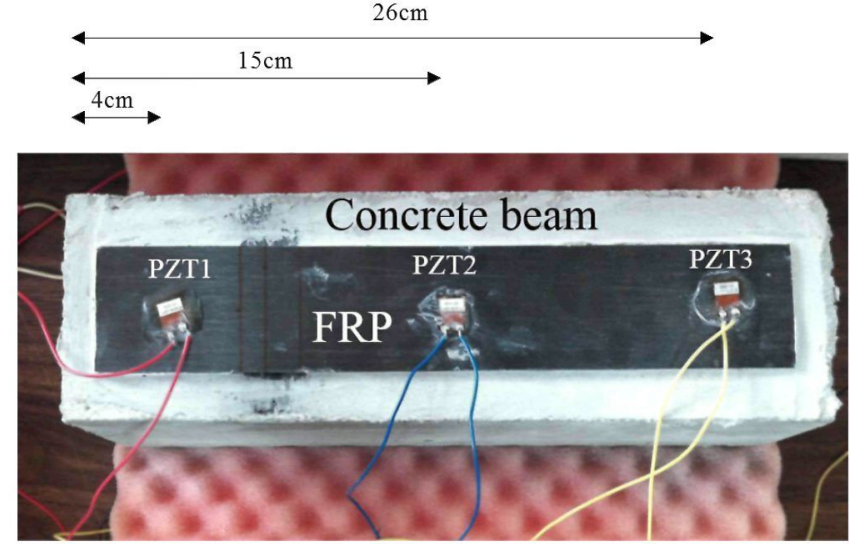

Fig. 6. Test specimen and the distribution of sensors.

the dimensions of the external reinforcement are $29 \mathrm{~cm}$ long, $5 \mathrm{~cm}$ wide and $0.12 \mathrm{~cm}$ thick. Three actuator-sensors were bonded to the external face of the FRP reinforcement using an epoxy adhesive. Their location was at a distance of $4 \mathrm{~cm}, 15 \mathrm{~cm}$ and $26 \mathrm{~cm}$, respectively, from the left side of the beam. The material properties of concrete, adhesive, FRP strip and PZT patch [43] are shown in Table 1. The three PZT sensors were individually connected to three different channels of a multiplexor, which was used to make a multiple connection between the HP 4192A Impedance Analyzer from Agilent Impedance Analyzer and the PZT sensors. The Impedance Analyzer excited the PZT sensors and simultaneously recorded the impedance signatures received by the PZT sensors. Captured data were stored in a personal computer. Fig. 7 shows the overall experimental setup.

To measure the impedances, a sinusoidal sweep voltage with an amplitude of 1 volt was applied to the PZT sensors over a frequency range between $10 \mathrm{kHz}$ and $100 \mathrm{kHz}$. Initially, a first test was performed on the specimen in its healthy condition. After measuring the baseline impedance signature, a hole type damage over a selected location was introduced at the FRP-concrete interface (Fig. 8) by using a drill. For each different stage, including the baseline and damaged conditions, a total of five impedance measurements were taken at different times on different days since some changes might appear in the impedance measurements due to slight changes in room temperature during the tests.

\subsection{Validation of the numerical impedance model}

Initially, the accuracy of the proposed numerical impedance model based on SEM was validated with the experimental impedance measurements obtained from the FRP-strengthened concrete block in healthy conditions. In SEM the mass of the structure is often referred to as being treated exactly; therefore,

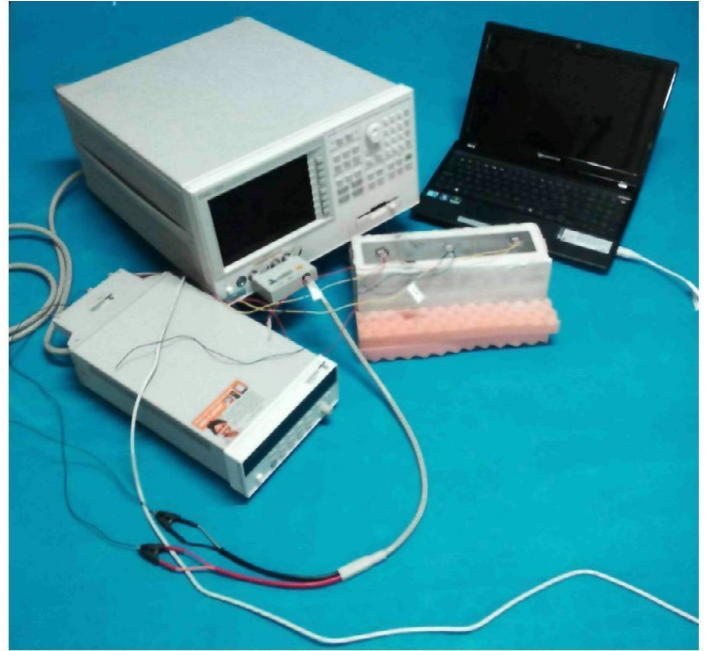

Fig. 7. Experimental setup.

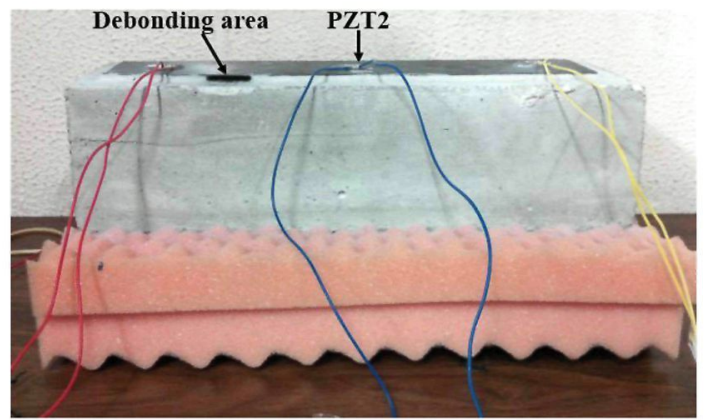

Fig. 8. Experimental test in FRP debonding scenario.

if there are no discontinuities, elements can span entire parts of the specimen for which the cross section remains unaltered. According to this, in healthy conditions, nine spectral elements were used to simulate the tested specimen (Fig. 9). Two elements correspond to the end non-strengthened portions of the beam [28], three elements based on the model proposed in Section 2 were used for the three strengthened portions of the beam with PZT bonded and, finally, the other four elements correspond to the strengthened portions of the beam without PZT [28]. The length of each one of these last four elements will depend on the location of the sensors.

Considering the load level introduced by the driving voltage from PZT, nonlinear behavior of the materials was not taken into account in the numerical model and elastic material properties were assumed to be distributed uniformly among all elements.

Table 1

Material and geometrical properties.

\begin{tabular}{|c|c|c|c|c|}
\hline Material properties & Concrete & Adhesive & FRP & PZT \\
\hline Elastic modulus $\left(\mathrm{N} / \mathrm{m}^{2}\right)$ & $28.58 \times 10^{10}$ & - & $16.5 \times 10^{10}$ & $6.21 \times 10^{10}$ \\
\hline Shear elastic modulus $\left(\mathrm{N} / \mathrm{m}^{2}\right)$ & $1.19 \times 10^{10}$ & $3.94 \times 10^{9}$ & $6.11 \times 10^{10}$ & - \\
\hline Length $(\mathrm{m})$ & 0.313 & 0.29 & 0.29 & 0.01 \\
\hline Width $(\mathrm{m})$ & 0.095 & 0.05 & 0.05 & 0.01 \\
\hline Thickness(m) & 0.075 & 0.001 & 0.0012 & 0.0002 \\
\hline Density $\left(\mathrm{kg} / \mathrm{m}^{3}\right)$ & 2400 & - & 1600 & - \\
\hline Piezoelectric constant ${ }_{31}(\mathrm{~V} / \mathrm{m})$ & - & - & - & $-8.30 \times 10^{8}$ \\
\hline Dielectric constant $\beta_{33}(\mathrm{mV} / \mathrm{C})$ & - & - & - & $7.42 \times 10^{7}$ \\
\hline Mechanical loss factor $\eta$ & - & - & - & 0.03 \\
\hline Dielectric loss factor $\delta$ & - & - & - & 0.02 \\
\hline
\end{tabular}




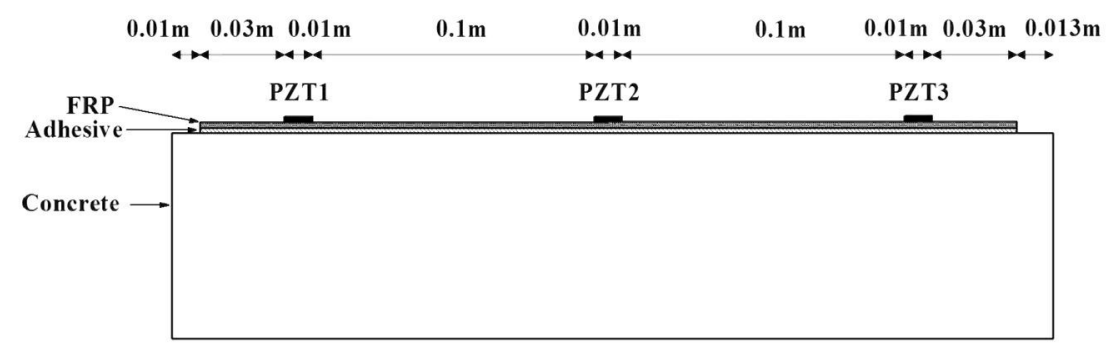

Fig. 9. Spectral element mesh for the undamaged specimen.

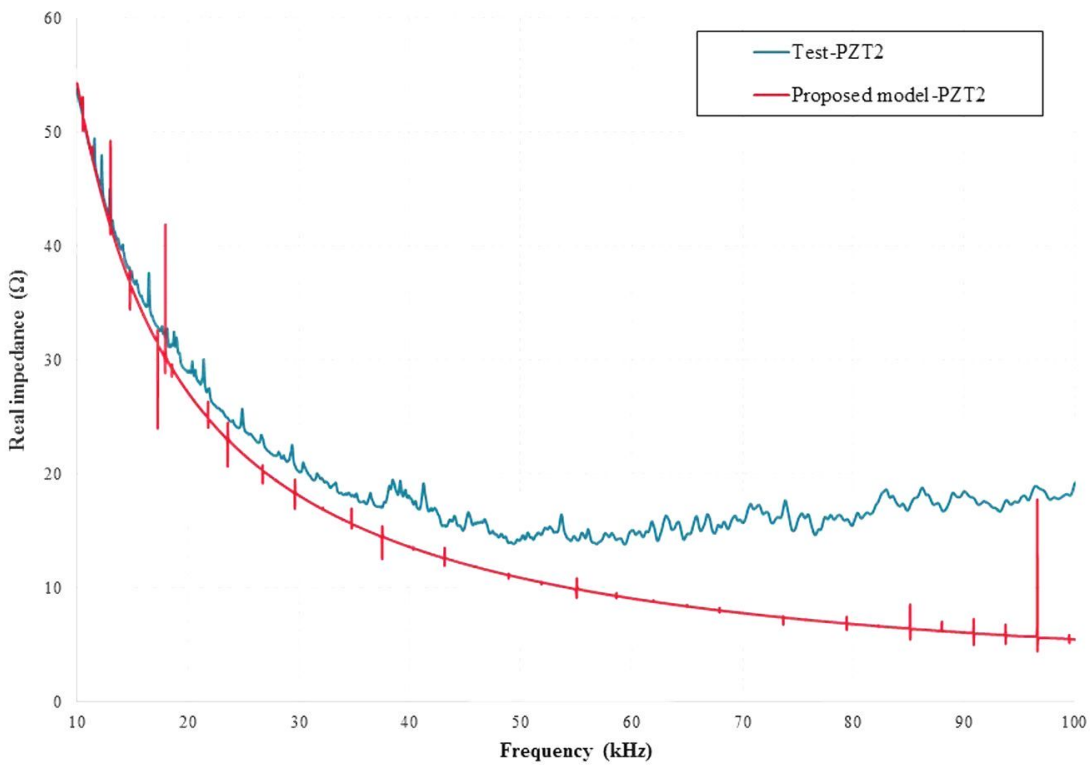

Fig. 10. Comparison of experimental and numerical impedance spectrum for an undamaged specimen (Real part).

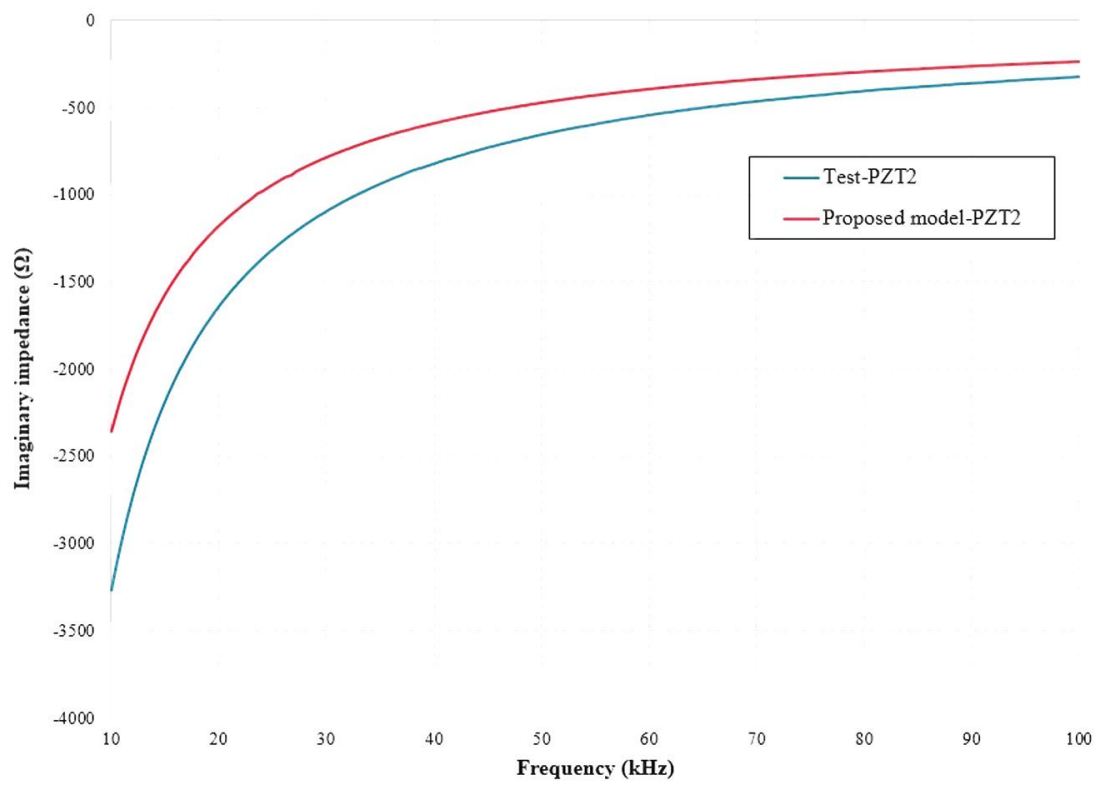

Fig. 11. Comparison of experimental and numerical impedance spectrum for an undamaged specimen (Imaginary part).

Although the material properties of PZT sensors might suffer changes due to environmental variations, the piezoelectric constants were assumed to remain unaltered since the temperature only experienced slight changes during the duration of the test.
Figs. 10 and 11 illustrate the comparison of EMI spectra for PZT sensor 2 in terms of the real part and the imaginary part, respectively, obtained by the experiment and the numerical model in the frequency range of $10-100 \mathrm{kHz}$ for the undamaged specimen. 
The results demonstrate that the proposed simulation could predict the EMI spectrum with a satisfactory approximation at a minimum cost since only nine spectral elements were used. As expected, some deviation of simulation data from experimental results appears which can be due to the assumptions carried out in the development of the model and to the uncertainties associated with the experimental tests. The deviation is higher for the imaginary part since this is more sensitive to temperature variation while the real part of impedance signatures is more sensitive to structural damage and other changes in structural properties and, as commented above, the effect of the temperature was not considered in the piezoelectric constants. For this reason, in damage detection the real part of impedance is usually employed.

\section{Damage identification results}

The experimental test on an FRP-strengthened concrete block presented in Section 5 was used to confirm the efficiency of the proposed damage detection approach. To do this, as commented above, a $2.4 \mathrm{~cm}$ length hole-type damage was introduced at the FRP-concrete interface at a distance of $7 \mathrm{~cm}$ from the left end of the concrete block (Fig. 8). Five impedance signatures were measured by sweeping a wide frequency band of $10-100 \mathrm{kHz}$, and the average values were computed. Fig. 12 shows a comparison of the real part of the undamaged and damaged impedance measurements for the second sensor. From their observation it is difficult to get some information about damage. To apply the proposed damage detection strategy, a very simple spectral numerical model was implemented originally for the specimen. For simplicity, for damage detection, sensor 3 was not used since it is located far from debonding area considering that we are working with high frequencies. Furthermore, some soldering failure made the measurements obtained with sensor 1 unusable. This is why only measurements from sensor 2 were applied in the damage identification procedure which was used to check the robustness and effectiveness of the proposed method considering that damage location is also one of its objectives.

Spectral element mesh for the configuration with the second sensor is illustrated in Fig. 13. The model consists of 7 spectral elements (E1-E7) and 8 nodes (N1-N8) whose coordinates were suitably chosen to be coincident with the sensor locations and the damaged area. Two elements, E1 and E7, were assigned to the end non-strengthened portions of the specimen and one element, E5, was assigned to represent the FRP-strengthened RC beam bonded with a PZT sensor by using the model derived in Section 2 . The remaining elements (E2-E4 and E6) were used in the FRPstrengthened portion of the concrete block clearly delimiting the damaged region, i.e. an element, E3, was chosen coincident with the debonding area. It is clear that the number of spectral elements used in the tested specimen to capture the high frequency impedance response is very low compared to the conventional finite element method. Intermediate debonding damage is studied in

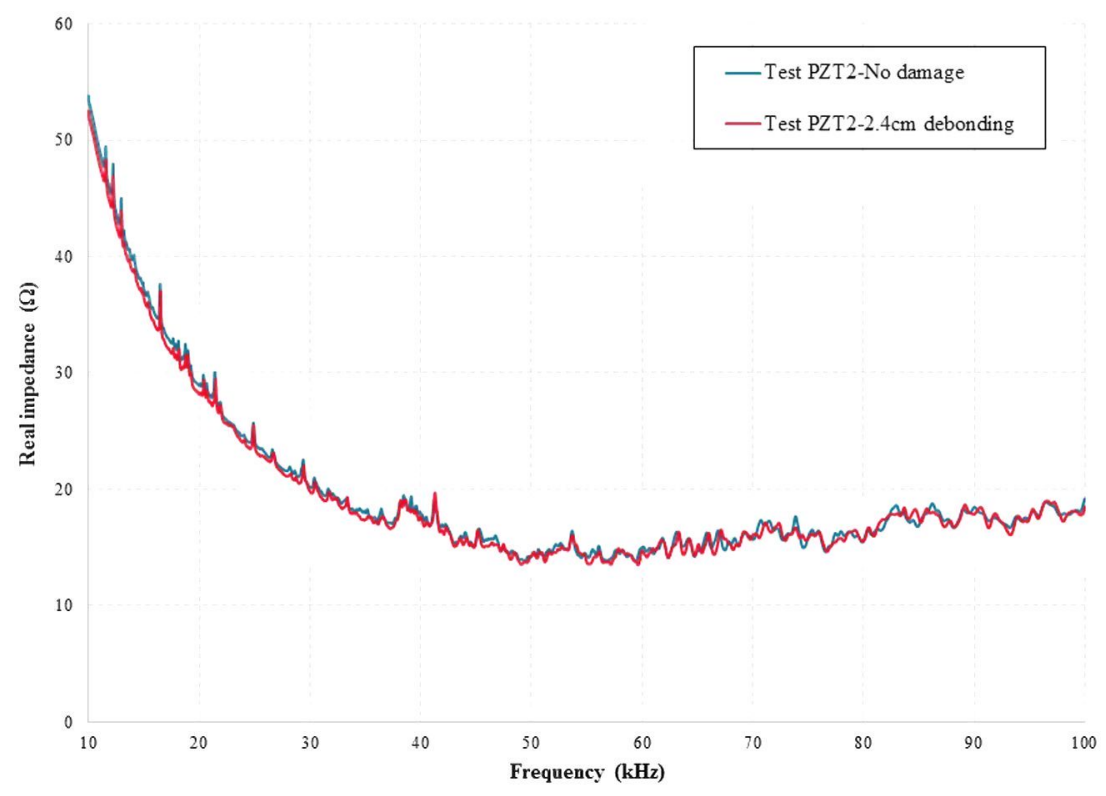

Fig. 12. Real part of impedance signatures captured from sensor 2 in undamaged and damaged conditions.

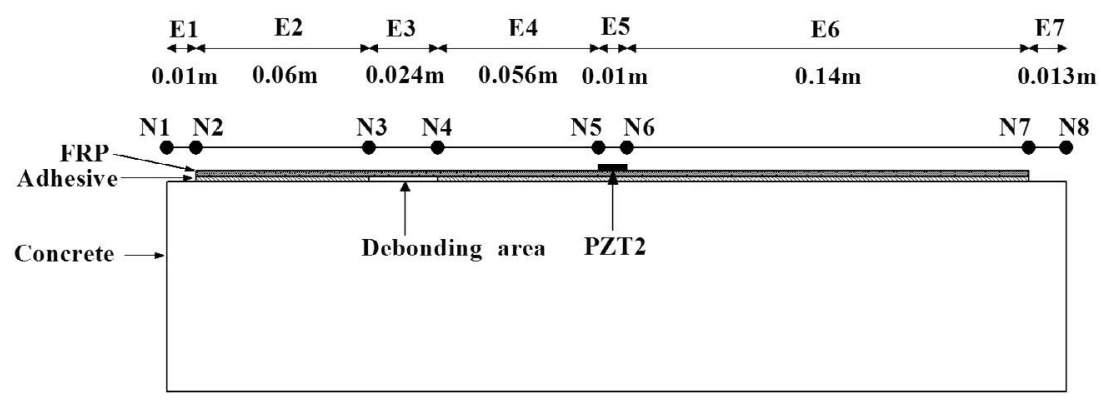

Fig. 13. Spectral element mesh for the configuration with PZT2. 


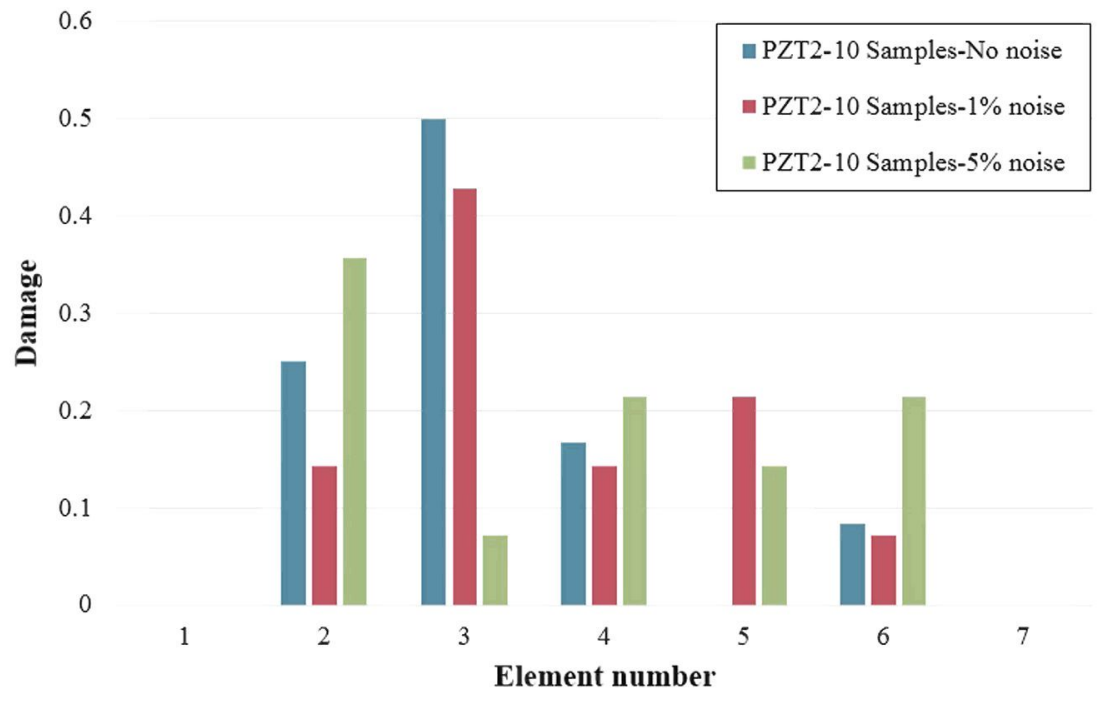

Fig. 14a. Damage distribution with PZT2 - 10 replicates.

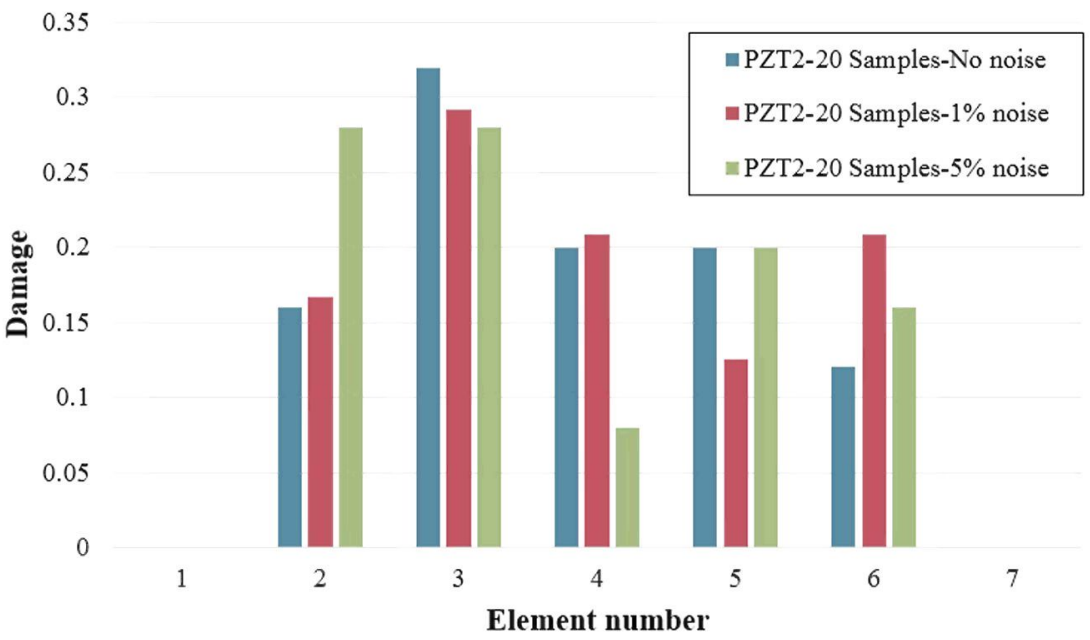

Fig. 14b. Damage distribution with PZT2 - 20 replicates.

this work. Therefore, damage will not occur at the two end nonstrengthened regions. According to Eq. (44) the damage vector will have only five components, one for each of the elements E2-E6.

Additionally, the impedance signals in a wide frequency range $(10-100 \mathrm{kHz})$ were divided into nine subranges with $10 \mathrm{kHz}$ each. Then, the CC vector defining the objective function in Eq. (43) was constructed with nine $\mathrm{CC}$ values calculated in nine sub-frequency ranges.

Another key point to be solved when the ensemble PSO algorithm is implemented is regarding the number of bootstrap replicates or samples to be applied. In a previous work [40], several tests on real and simulated data sets were carried out and it was found that using only 10 bootstrap replicates can lead to an improvement in accuracy but an increasing number of samples might reasonably improve the classification accuracy. For this reason, in this work, three different data sets of 10,20 and 30 were tested to decide the final damage prediction. For each PSO learning machine, a random and replicate initial population of 10 individuals was created and twenty simulation runs were performed. Each machine will predict one element as damaged and vote for it; the element predicted most often by the sub-classifiers or machines will be chosen as the damaged element. Furthermore, the severity of the voted elements by each machine will also be considered.

To carry out the study, three different scenarios were considered, unnoisy, $1 \%$ and $5 \%$ of noise. In the experimental tests, measurements are subject to external uncertainties, even if the test is well-controlled within a laboratory setting. To be more severe and check the robustness of the method, we also contaminated the lab data obtained from sensors with artificial noise using two different levels of severity, $1 \%$ and $5 \%$.

Figs. $14 a-c$ show the identification results for the three noise cases by using 10, 20 and 30 bootstrap samples, respectively. Predictions with $1 \%$ noise are good and are very similar to the unnoisy case; in these two cases predictions are not affected by the number of samples used. However, when an artificial noise of $5 \%$ is introduced, the identification of the damaged element becomes impossible when 10 and 20 samples are used. In this case, changes introduced in the impedances by the level of noise are higher than the changes due to the appearance of the debonding defect. However, it is very interesting to notice that in Fig. $14 \mathrm{cc}$ the damage prediction is not so influenced by the effect of $5 \%$ noise since the number of PSO reaches 30 , which shows that the stability of prediction increases with the number of PSO in noisy conditions. 


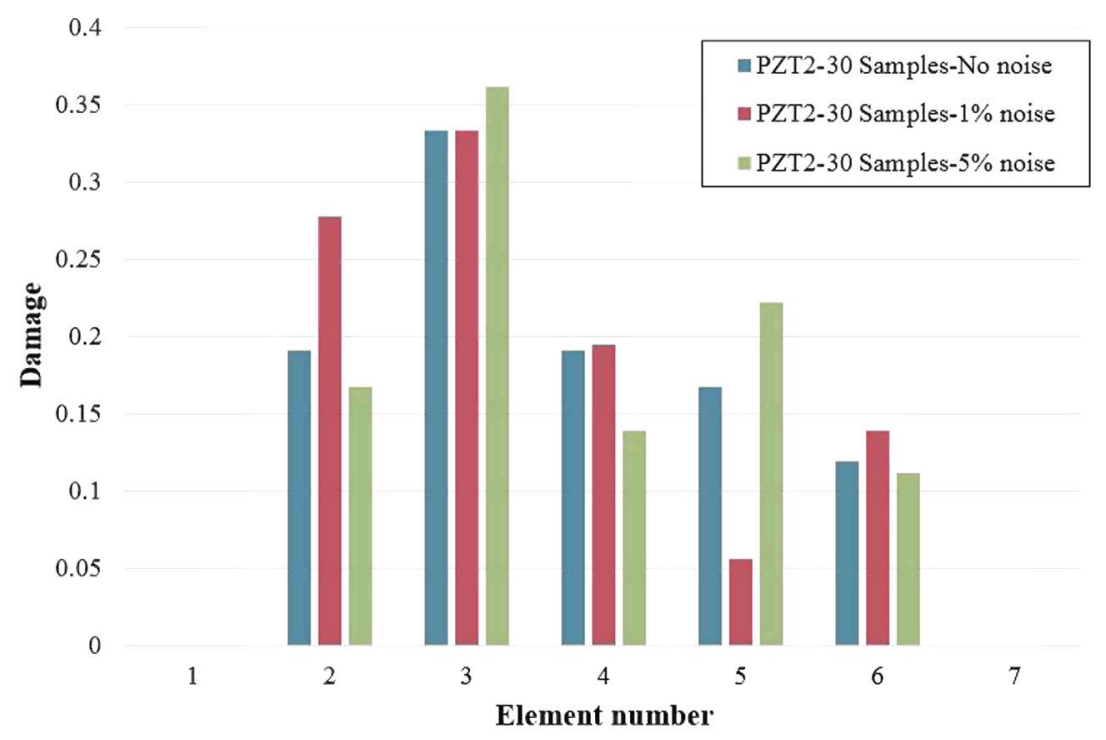

Fig. 14c. Damage distribution of PZT2 - 30 replicates.

\subsection{Adaptive mesh}

A suitable method to monitor the health of concrete structures repaired and reinforced with composite material will require not only the detection of disbonds and their severity but also locating them. In the previous study, the spectral element mesh shown in Fig.13 was adapted to the debonded region in such a way that one element was always chosen fully coincident with this region. Therefore, if this element is identified as damaged, the location of disbond agrees with the position of the element. However, in real practical applications it is clear that damaged areas will not agree exactly in size with elements of the numerical model and, therefore, the effect of mismatched element size might affect the identification of the damage location. To avoid this, an updating procedure based on an adaptive mesh has been developed in this work.

To explain the procedure, consider firstly the mesh shown in Fig. 15 formed by nine spectral elements and ten nodes distributed along the length of the FRP strengthened beam. The position of nodes $1,2,9$ and 10 will always remain unaltered since these nodes delimit the non-strengthened portion of the concrete specimen. The same occurs with nodes 5 and 6 since these nodes agree with the location of the PZT layer. Nodes 3 and 4 on the left of the sensor and nodes 7 and 8 on the right of the sensor will be used to identify the location of the damaged area and, therefore, their position will change along the procedure. This will be based on the use of an adaptive mesh which will move towards the debonded area. An outline of the complete procedure to determine the location of the damaged area is as follows:

\section{(a) Initialization}

The coordinates of nodes $3,4,7$ and 8 will be generated randomly and the methodology of damage identification, previously presented, based on ensemble PSO will be applied. An element will be assigned as damaged.

(b) Iterative solution of the problem (Iteration $m$ ) The purpose of the proposed updating strategy is to let nodes 3 and 4 approach the damage location as long as damage exists on the left side of the specimen or, similarly, do the same with nodes 7 and 8 if damage is detected on the right side of the specimen. To do so, according to the detec- tion results obtained in the previous iteration, the coordinates of nodes $3,4,7$ and 8 will be updated by using the following algorithm:

If element assigned as damaged is on the left side of the specimen (elements E2, E3 or E4)

update the coordinates of nodes 3 and 4 : $x 3(m+1)=x 3(m)-\left(x 3(m)-d a m_{-} l o c\right) / 2$ $x 4(m+1)=x 4(m)-(x 4(m)-$ dam_loc $) / 2$ coordinates of nodes 7 and 8 remain unaltered

else if element assigned as damaged in $E 5$

coordinates of nodes 3, 4, 7 and 8 remain unaltered

else if element assigned as damaged is on the right side of the specimen (elements E6, E7 or E8)

coordinates of nodes 3 and 4 remain unaltered

update the coordinates of nodes 7 and 8 : $x 7(m+1)=x 7(m)-(x 7(m)-$ dam_loc $) / 2$ $x 8(m+1)=x 8(m)-\left(x 8(m)-d a m_{-} l o c\right) / 2$

In this algorithm dam_loc represents the location of the left node in the element assigned as damaged.

Once the coordinates have been updated, the methodology of damage identification based on ensemble PSO is applied again.

(c) Convergence criterion

After each iteration, the convergence of the procedure must be verified. To do this, the solution of the procedure can be accepted when no significant differences in coordinates arise between the $m$ th iteration and the former one.

The proposed adaptive algorithm has been used to identify the damage location of the specimen shown in Fig. 8 from experimental impedances captured in sensor 2. Fig. 16 shows the evolution of the element assigned as damaged by the algorithm along the iterative procedure. It can be observed that when the iteration number exceeds 11 , the element identified as damaged converges to element 3 which is between nodes 3 and 4; this means that the location of nodes 3 and 4 will delimit the location of the debonded area. On the other hand, Fig. 17 shows the iterative evolution of the coordinates of nodes 3, 4, 7 and 8 . As illustrated in this figure, the 


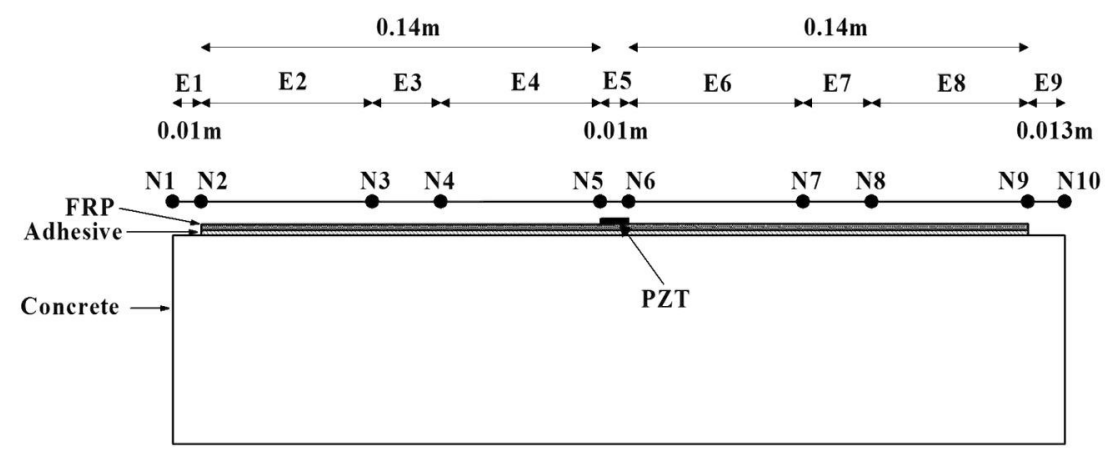

Fig. 15. Adaptive spectral element mesh for damage location.

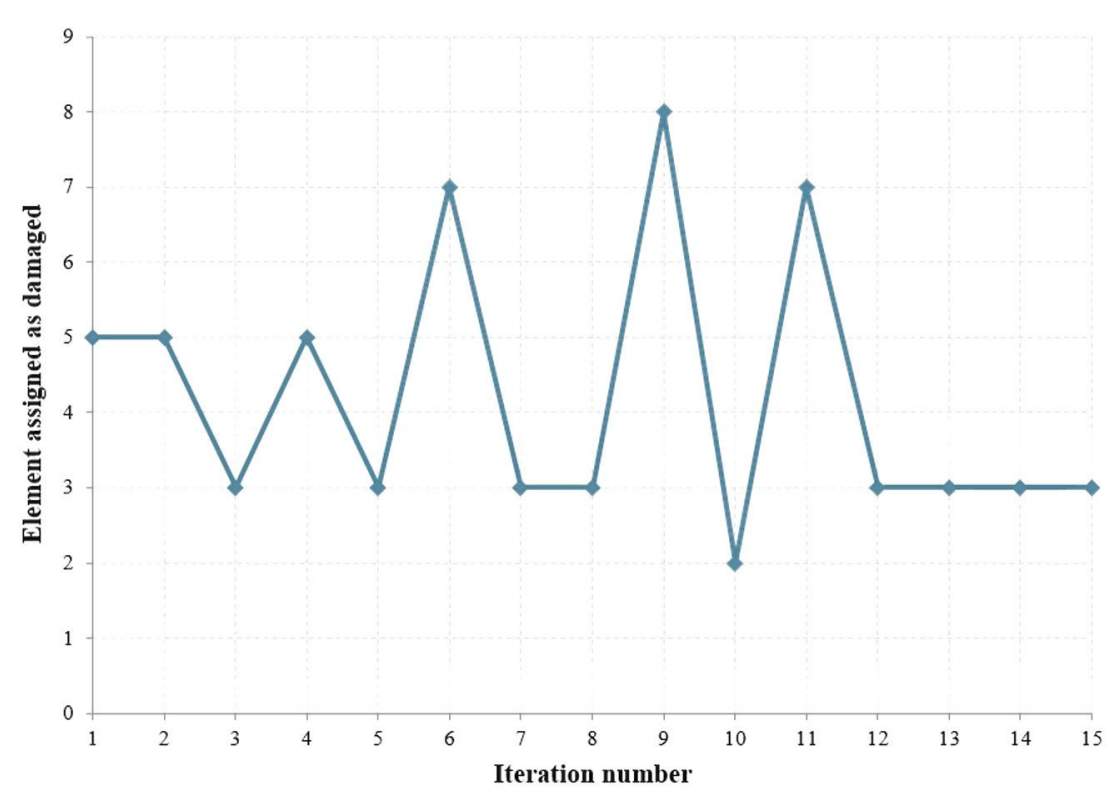

Fig. 16. Evolution of the element assigned as damaged by the adaptive algorithm.

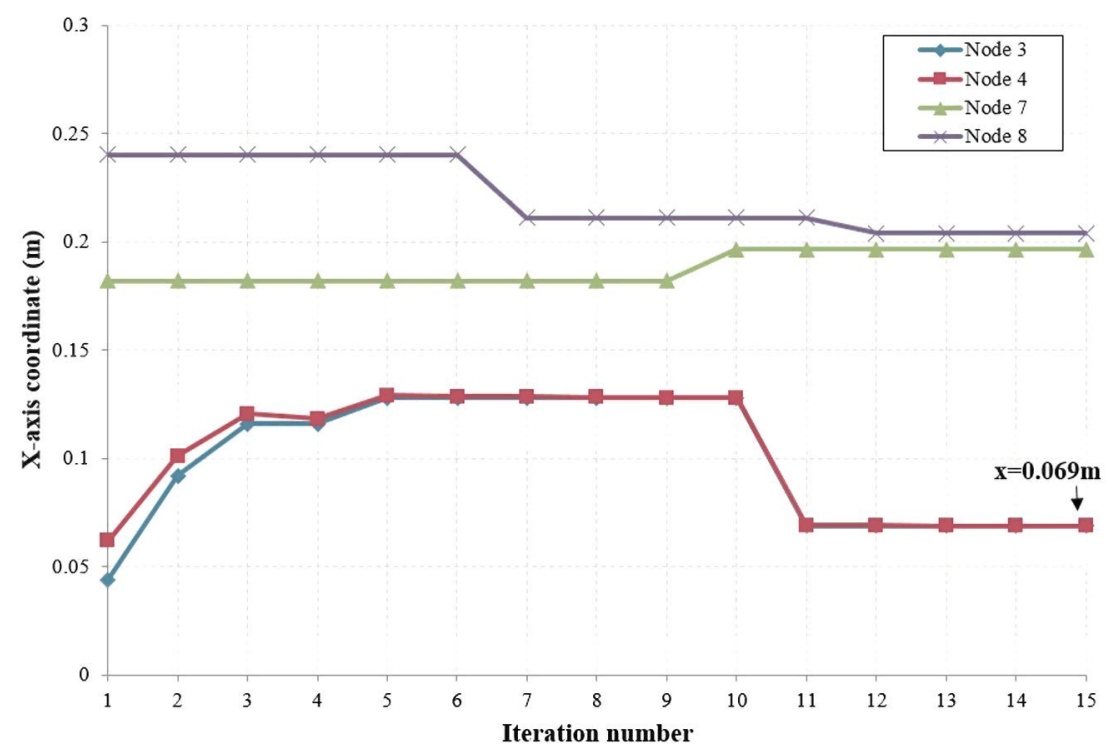

Fig. 17. Evolution of nodal coordinates during the iterative procedure.

coordinates of nodes 3 and 4, belonging to element E3 assigned as damaged, become practically coincident when the convergence of the iterative procedure is reached. Furthermore, their value is
$0.07 \mathrm{~m}$ while in the tested specimen the location of the debonded area is at a distance of $0.07 \mathrm{~m}$ from the left end of the specimen This demonstrates the accuracy of the proposed adaptive updating 
strategy based on ensemble PSO as a tool to identify minor debonding in FRP-strengthened RC beams in both aspects of severity and location.

\section{Conclusions}

A methodology to diagnose damage due to intermediate interfacial debonding in FRP flexural strengthened RC beams has been proposed. The approach is based on a PZT impedance actuatorsensor. These kinds of transducers works in the high frequency range which makes them especially suitable to detect minor debondings. The adopted strategy is based on a model updating procedure and has been carried out with a proposed model of the bonded-PZT FRP strengthened beam based on a one-dimensional simplified spectral element method which allows working easily in the high frequency range in a non-expensive way. Considering the local nature of the studied damage, its identification represents a challenging task. With the purpose of increasing the efficiency and robustness of the proposed methodology two additional contributions have been carried out. Firstly, an ensemble method based on the standard PSO algorithm has been implemented. Secondly, an adaptive algorithm has been developed to increase the performance of the proposed strategy with respect to the identification of the damage location in real applications.

An experimental study was performed to validate the proposed scheme. Successful predictions have demonstrated the importance of the proposed contributions to improve the capabilities of damage identification in both severity and location. Because of this, it appears to be very promising as an active non-destructive evaluation technique. In future studies, the proposed approach will be checked on RC beams strengthened with FRP strips during a loading procedure until failure.

\section{Acknowledgements}

The writers acknowledge the support for the work reported in this paper from the Spanish Ministry of Economy and Competitiveness (project BIA2013-46944-C2-1-P). Financial support for the CSC research fellowship given to Rui Sun and the FPI research fellowship given to Enrique Sevillano are also acknowledged. 